\title{
SIMULATION OF THE TEXTURE EVOLUTION DURING ANNEALING OF COLD ROLLED BCC AND FCC METALS USING A CELLULAR AUTOMATION APPROACH
}

\author{
V. MARX, D. RAABE, O. ENGLER and G. GOTTSTEIN \\ Institut für Metallkunde und Metallphysik, \\ SFB 370 "Integrative Werkstoffmodellierung" der DFG, \\ Kopernikusstr. 14, RWTH Aachen, 52056 Aachen, Germany
}

(Received 18 April 1996)

\begin{abstract}
In this study both primary static recrystallization and static recovery of cold rolled bcc and fcc metals and alloys are numerically simulated using a three-dimensional model that is based on a modified cellular automaton approach. The model considers the influence of the initial deformation texture and microstructure on both static recovery and primary static recrystallization with a high spatial resolution. The cellular automaton technique provides both local and statistical information about the kinetics, the morphology and the texture change during annealing. The influence of nucleation and growth can be studied in detail. The simulations are compared to experimental results obtained on fcc and bcc polycrystals.
\end{abstract}

KEY WORDS: Recrystallization recovery deformation texture microstructure cellular automaton nucleation growth kinetics.

\section{INTRODUCTION}

The mechanical properties of polycrystalline metallic materials are mainly determined by their microstructure and crystallographic orientation distribution. The latter one is widely referred to as crystallographic texture. Thus it is highly important to understand the physical processes which determine the development of these parameters in order to get insight into the evolution of the properties of the materials. Prior to any prediction of the structural evolution a detailed microstructural description of the material under investigation is required. Further experimental data about the course of recrystallization are pertinent for critically evaluating the simulation.

The processes of deformation, recovery and recrystallization have been under thorough experimental examination since the beginning of systematic materials research. The experimental methods of acquiring data on the corresponding materials characteristics have been refined over the years. Additionally, during the last decades the techniques for the simulation of deformation processes have been improved to a degree, that makes them useful for theoretical and practical application. Despite this abundance of reliable data on deformation (experimental, simulation) and annealing processes (experimental), there is still a substantial lack of corresponding simulations in the field of recovery and recrystallisation. 
Using this broad range of data it is now possible to simulate the recrystallization of heavily deformed materials in its entirety. Starting from the deformed microstructure the process can be studied in a detailed manner using computer experiments. Rigorous verification of these simulations can always be achieved relying only on experimental data. Furthermore, convergence with analytical approaches can be easily checked for behaviour at the limit of the model. Thus, one may expect to gain substantial insight into the physical processes during the annealing of heavily deformed metals.

After verification, the employment of computer experiments can be used to optimise the material properties. The primary static recrystallization is the most important process during the heat treatment of heavily deformed metals. It is necessary to understand the development of the crystallographic texture during this process. Thus it is possible to gain the ability to predict and optimise the mechanical behaviour of the specimen under consideration. The present study describes a model for the simulation of primary static recrystallisation and static recovery. Two typical applications are presented and discussed.

\section{DESCRIPTION OF THE MODEL}

The model for the simulation of primary static recrystallization is based on a cellular automaton approach. The model considers the local microstructure and microtexture at length scales ranging from a few subgrains up to several hundred grains. From the numerical simulation, one obtains data about the kinetics, the microstructural and textural evolution during annealing of cold worked metals and alloys. It is possible to cope with largely different nucleation and growth conditions ranging from pure recovery to dynamic recrystallization. The model incorporates both nucleation and the subsequent growth of the nuclei into the deformed matrix.

At the start of the simulation, a cubic lattice is superimposed on a representative portion of the deformation microstructure. Each cell in this lattice is characterised through its orientation, its dislocation density and its site. The state of a cell $x$ at the time $t_{i}$ is determined through the state of its neighbour at the previous timestep $t_{i-1}$. A great variety of possible transition rules exists. This leads to a good adaptability of the model to other cases. Cyclic boundary conditions are assumed for the lattice. The model is discrete both in time and space.

For the simulation of recrystallization two steps are considered: The first step regards the formation of nuclei inside the deformed matrix. The second step treats their subsequent growth into the deformed matrix. To simulate nucleation, spheres of a given radius are arranged within the simulated volume. The employment of spheres as nuclei is necessary to overcome the influence of the grid geometry on the grain geometry. The dislocation density of all cells within these spheres is decreased to the dislocation density of the non-deformed matrix. To save computer memory only cells belonging to the surface of these nuclei are considered during the subsequent computations. The crystallographic orientation distribution of the nuclei is determined by random selection out of a predefined nucleation spectrum. This rule represents the case of oriented nucleation. By variation of the employed probability matrix, any kind of oriented nucleation can be incorporated. Furthermore, it is possible to vary the nucleation condition temporal, e.g. site saturation or constant nucleation rate. Furthermore, a spatial variation, e.g. homogeneous nucleation, nucleation at grain boundaries, nucleation at deformation inhomogineities can be introduced into the model. 
Growth of the nuclei into the surrounding deformation matrix is simulated by a displacement of the cells belonging to their surface. The direction of growth is the normal direction of the surface element taken into consideration. The growth velocity depends on the driving force and the grain boundary mobility. Both physical characteristics are entirely independent. The former property is defined through the dislocation density of the deformed matrix. The dislocation arrangement is not yet considered. The latter property is defined in terms of the misorientation between a growing nucleus and the surrounding matrix. All cells in the surface of a growing nucleus are displaced into the surrounding matrix according to their growth velocity and form the new surface of the nucleus. All non-recrystallized cells that are encountered by the moving surface are marked as being recrystallized.

Upon impingement of two recrystallized grains the growth of these nuclei stops. The simulation is stopped, when the entire matrix consists of recrystallized cells. However, in case of strong competitive recovery it is conceivable, that primary recrystallization comes to an end, although some areas of the deformation matrix have not been removed by moving large angle grain boundaries. Post-processing of the simulation data yields information on texture, grain size distribution, microstructure and kinetics.

The texture description is accomplished through the use of model components. The starting texture is discretised using isotropic scattering model components (Lücke et al., 1981). The grains inside the simulated microstructure are correlated with the texture components in a manner that preserves the volume fraction of these components. Typically about 10 texture components are used for the description of the crystallographic texture. The resulting texture is determined through the volume fractions of the different texture components. It is displayed by means of a Gauss type orientation distribution function (ODF) (Lücke et al., 1981). The scatter width of the isotropic Gauss type components has a strong influence on the quantitative value of the ODF. This value is assumed to be $10^{\circ}$ for the description of ODFs after recrystallisation, to compensate for the limitation to only a few texture components. Still, the sharpness of the displayed ODFs resulting from simulation tends to be too high when compared to experimental results. Therefore, we mainly concentrate on a qualitative comparison between experimental textures and those obtained from simulation.

\section{RESULTS AND DISCUSSION}

\section{Nucleation of Recrystallisation in Aluminium Alloys}

One of the problems to which this simulation was applied is the texture development during the recrystallization of aluminium alloys. During the investigation of recrystallization in a two-phase $\mathrm{Al}-1.8 \% \mathrm{Cu}$ alloy Engler (1995) found an influence of the initial grain size, i.e. the grain size before cold rolling, on the final recrystallization texture. In the experiments conducted, it was possible to distinguish between two possible nucleation sites. First, nucleation of cube oriented grains can take place at band-like structures comprising mainly orientations near the cube orientation, the so called cube-bands. Additionally, particle stimulated nucleation (PSN) takes place throughout the rest of the matrix. The resulting recrystallisation texture can be interpreted as a superposition of the textures of cube-band nucleated grains and grains that stem from PSN. Since the initial grain size is correlated with the distance between the cube-bands, the influence of this grain size on the resulting texture can be described in terms of 
a simple model (Figure 1). In this model it is assumed that nuclei which stem from the cube-bands solely show the cube orientation, whereas nuclei forming by PSN show a random texture. This random texture is transformed through the process of growth selection into a texture which resembles the $40^{\circ}<111>$ rotated rolling texture. Experimental results for narrow and wide spaced cube-bands are shown in Figure 2a and $b$ respectively.

This model was incorporated into the nucleation procedure of the simulation. Small bands inside the simulated space were assumed to nucleate only cube oriented grains. In other parts of the matrix the crystallographic orientation of the nuclei was determined by random. The results are shown in Figure 3. Figure 3a shows the results for wide spaced cube-bands. One can clearly see a texture as would be expected from random nucleation and subsequent growth selection. However if the spacing of the cube-bands becomes more narrow, a higher amount of the cube orientation in the texture is evident (Figure 3b). Thus, the simulation using the aforementioned model yields results very similar to those observed experimentally (Figure 2).

\section{Recrystallisation and Recovery in Tantalum}

In bcc alloys we are faced with a totally different situation when compared to fcc metals. In these materials static recovery is known to play a major role in their textural evolution. Static recovery leads to a decrease in dislocation density inside a grain without discontinuously changing its crystallographic orientation. This can lead to residue of deformation texture components after annealing.

\section{Cube PSN}

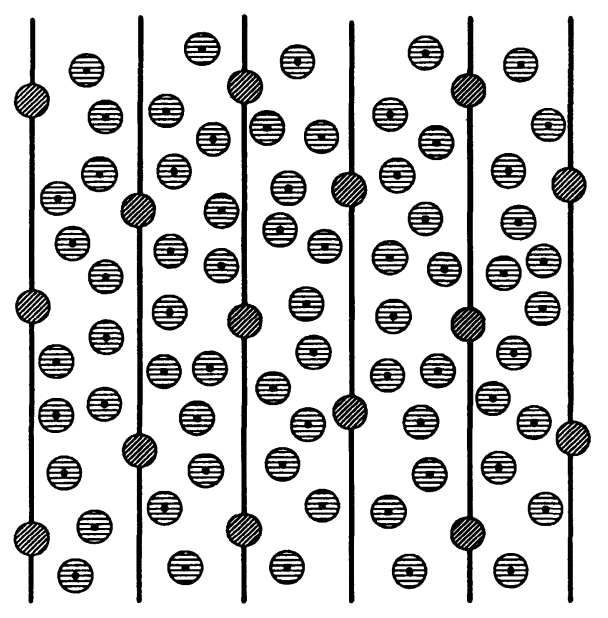

(a) Fine grained

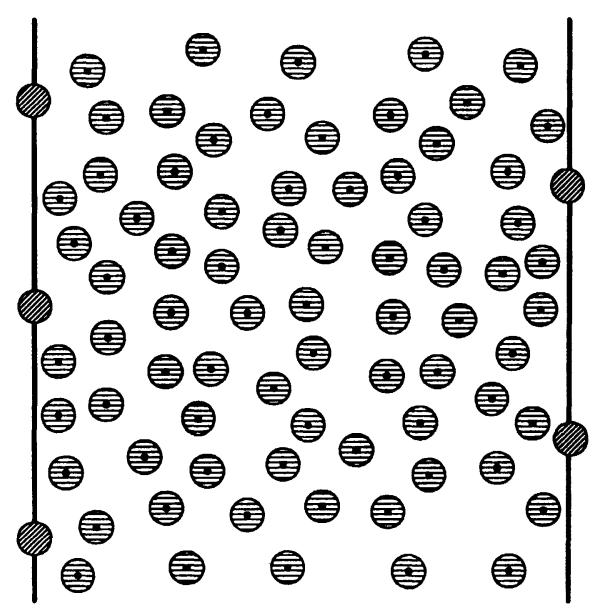

(b) Coarse grained

Figure 1 Schematic sketch of the spatial distribution of cube- and PSN-grains used in the model. (a) fine grained material, (b) coarse grained material. 

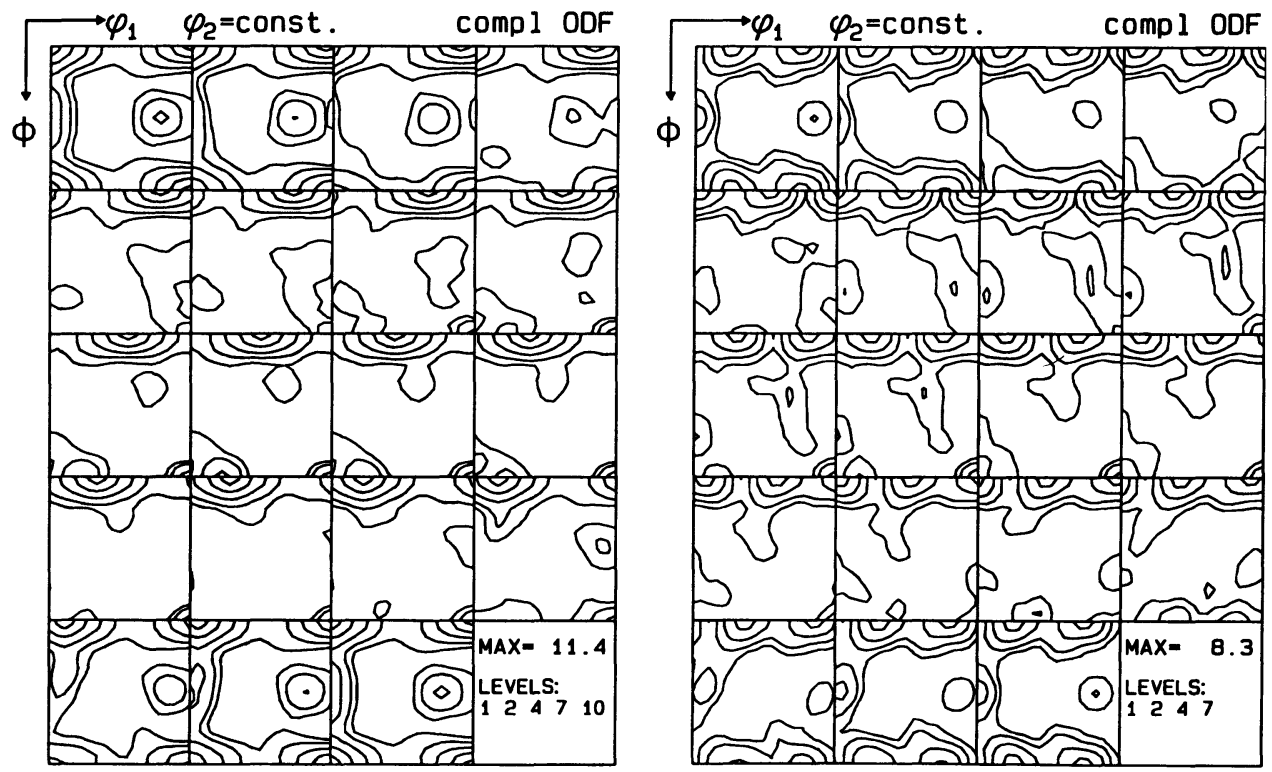

Figure 2 ODFs of the recrystallisation textures of an overaged, $90 \%$ cold rolled $\mathrm{Al}-1.8 \% \mathrm{Cu}$ alloy. (a) fine grained material, (b) coarse grained material.
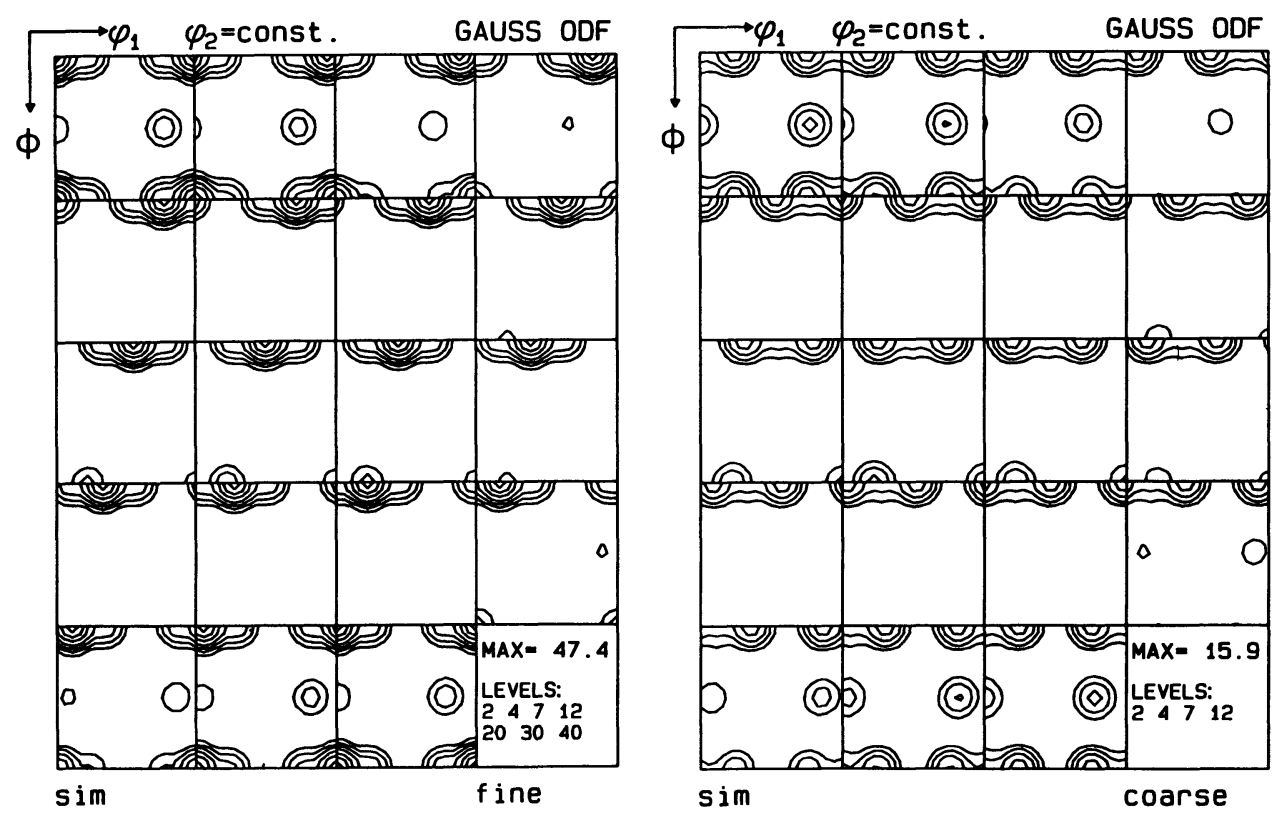

Figure 3 ODFs of the recrystallisation textures obtained by simulation for an $\mathrm{Al}-1.8 \% \mathrm{Cu}$ alloy. (a) fine grained material, (b) coarse grained material. 
Thus, for the simulation of annealing of cold deformed bcc alloys the cellular automaton simulation was complemented by a simulation of static recovery. For the simulation of static recovery we assumed a degradation of dislocation density in all non-recrystallized cells as a function of the annealing time.

$$
\rho(t)=\rho_{0} \cdot \exp \left(-\frac{t}{t_{0}}\right)
$$

This decreasing dislocation density leads to a change in the driving force and thus to a lower grain boundary velocity. If the dislocation density inside a simulation cell reaches a critical lower value, i.e. a dislocation density which does not allow further movement of the grain boundary, the corresponding area is identified as being recovered below this critical value. The simulation is terminated when all cells within the grid are either recrystallized or recovered.

The spatial limitation of the model requires an upper ceiling for the maximum displacement distance during one step of the model. In order to retain this limit and to simulate the different kinetics connected with recovery and recrystallization, we incorporated an adaptability of the time increments. The time increment that is associated with one step of the model is determined by the maximum displacement and the maximum grain boundary velocity during this step. The calculation of recovery kinetics takes into account the sum of all time increments.

As a model substance for bcc metals and alloys pure tantalum was chosen in the present study. Tantalum does not show phase transformation between room temperature and its melting point. For the simulation of the microstructural evolution during annealing a grid consisting of $100^{3}$ cells with periodic boundary conditions was used. The initial microstructure consisted of 30 deformed grains scaled by the above mentioned grid. The crystallographic orientations of these grains represent the texture of a cold rolled pure tantalum specimen (Figure 4). In the present study oriented nucleation was held responsible for the development of the recrystallisation texture. Nucleation was assumed to be site saturated and spatially random. The simulation results of calculations without incorporation of the recovery model show good accord with experimental results (Figure 5). The recrystallized specimen shows a strong so called $\gamma$-fibre, $\{111\}<110\rangle$ - $\{111\}<112>$, already weakly present in the cold deformed condition. The $\alpha$-fibre, $\{001\}<110>-\{111\}<110>$, strongly present in the cold rolled specimen, vanished completely.

The incorporation of recovery into the annealing model showed deviating results. Figure 6a shows the crystallographic texture of a simulated microstructure, consisting of $70 \%$ recrystallized and $30 \%$ recovered cells. This texture shows the strong $\gamma$ fibre, which is typical for recrystallized bcc alloys accompanied by a weaker $\alpha$-fibre normally associated with deformed bcc alloys. Since no discontinuous orientation change takes place during static recovery, the $\alpha$-fibre can be associated with the recovered deformation matrix, whereas the $\gamma$-fibre stems from the recrystallised cells. This type of texture compares well to a pure tantalum sample (Figure $6 \mathrm{~b}$ ) that was $90 \%$ cold rolled and annealed at $1000^{\circ} \mathrm{C}$, slightly lower than the fully recrystallized sample (Figure $5 \mathrm{~b}$ ). This result can be explained in terms of the strongly different kinetics of recrystallization and recovery. 

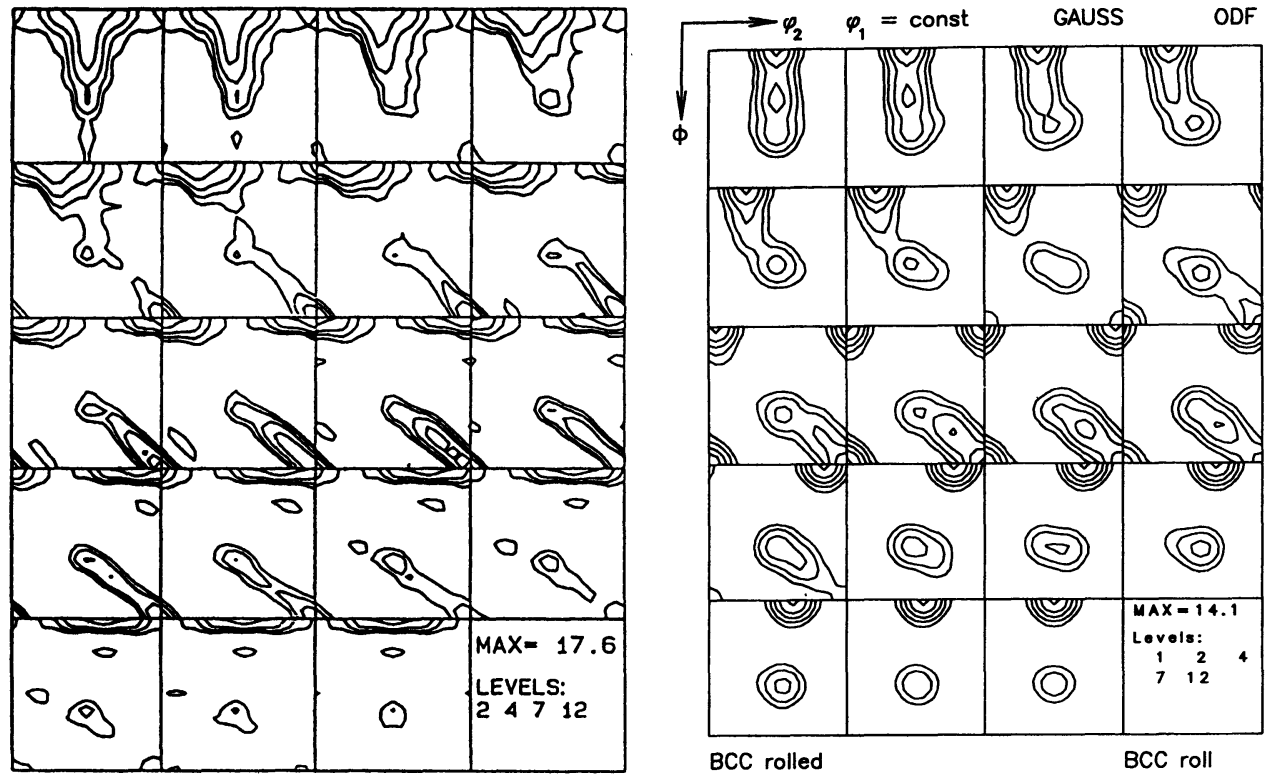

Figure 4 (a) Rolling texture in the centre layer of a $90 \%$ cold rolled pure tantalum specimen. (b) Representation of this texture as used as starting texture for the simulation.
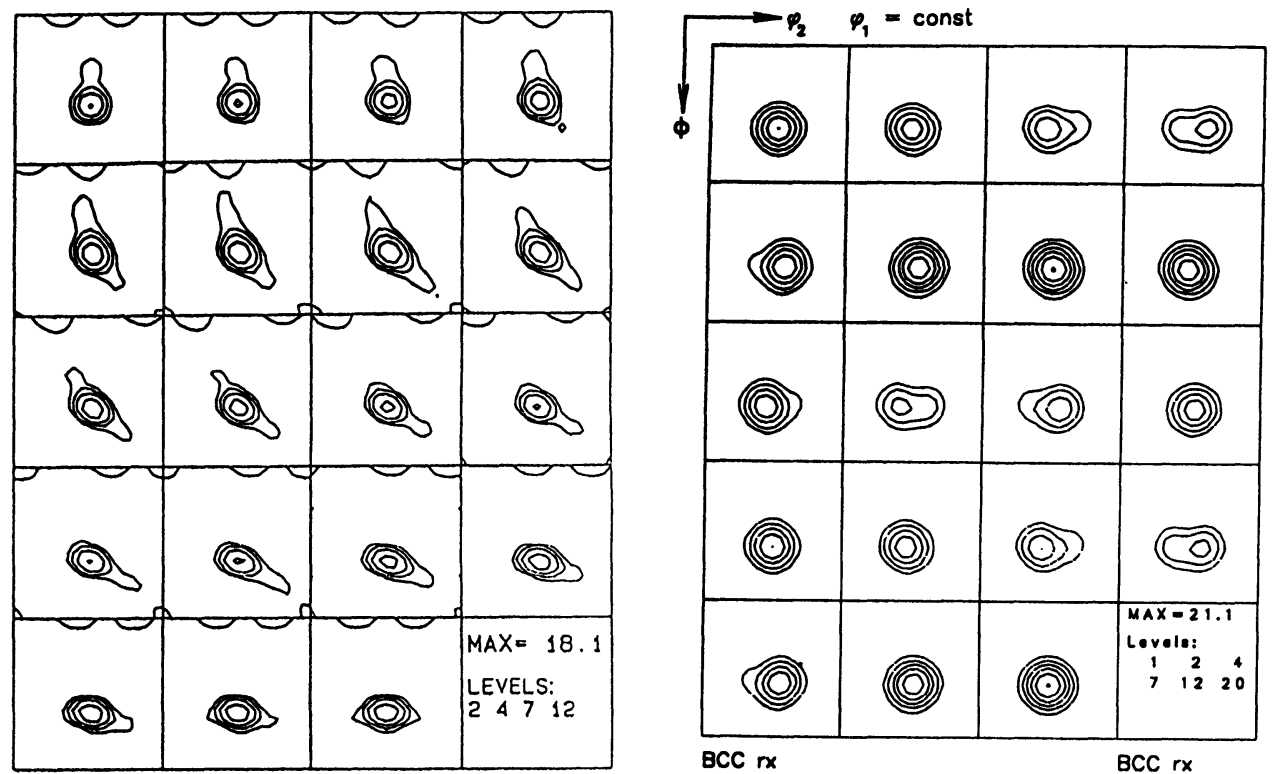

Figure 5 (a) Annealing texture of a pure tantalum specimen, $90 \%$ rolled, $1100^{\circ} \mathrm{C}$, $1 \mathrm{~h}$. (b) Texture of simulated microstructure after recrystallisation. 

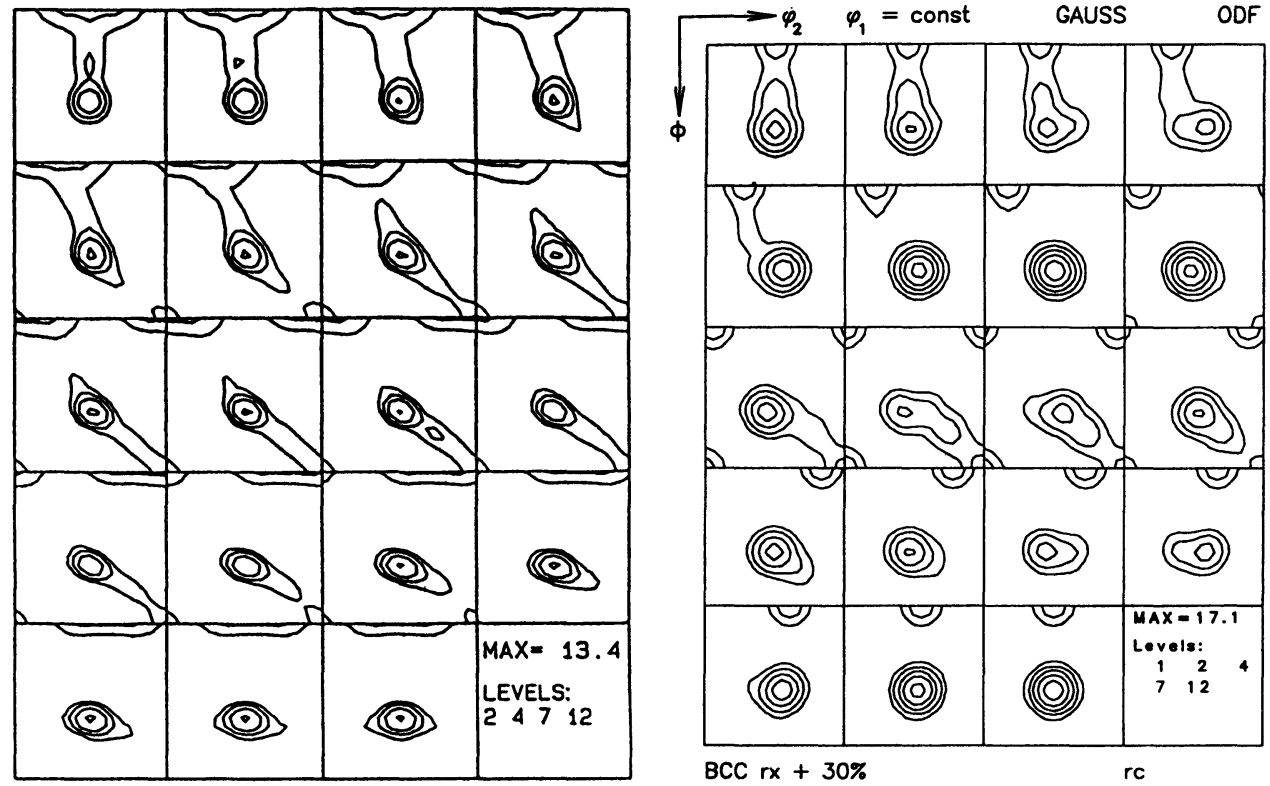

Figure 6 (a) Annealing texture of a pure tantalum specimen, $90 \%$ rolled, $1000^{\circ} \mathrm{C}, 1 \mathrm{~h}$. (b) Texture of simulated microstructure with $70 \%$ recrystallised and $30 \%$ recovered grains.

\section{SUMMARY}

It was shown that primary static recrystallization can be simulated by means of a threedimensional cellular automaton model. The adaptability of this model to a wide range of cases with a given deformation texture and microstructure was demonstrated. Additionally the simulation of static recovery was considered within this model. In the present study two widely different applications for the model were displayed.

Annealing processes in fcc and bcc alloys were numerically simulated. The calculations were performed assuming different sets of conditions for nucleation and growth of the nuclei. The simulations took into account the crystallographic texture, the local misorientation and the local dislocation density. The results of the simulation were in very good accord with experiment.

\section{Acknowledgements}

This research was supported by the Deutsche Forschungsgemeinschaft (DFG) through the Collaborative Research Centre 370 "Integrative Werkstoffmodellierung".

\section{References}

Engler, O. (1995). Influence of the initial grain size on the rolling and recrystallization textures in the alloy $\mathrm{Al}-1.8 \% \mathrm{Cu}$. Textures and Microstructures, 23, 61-86.

Lücke, K., Pospiech, J., Virnich, K. H., and Jura, J. (1981). On the problem of the reproduction of the true orientation distribution from pole figures. Acta Met., 29, 167-185. 